\title{
Dependable Wireless Sensors Module Hardware Design for Smart Universities Application
}

\author{
Yasser M. Alharbi ${ }^{1}$, Mohamed S. Soliman ${ }^{1,2 *}$, Farhan A. Salem ${ }^{3}$, Ahmad A. Alahmadi ${ }^{1}$, \\ H. Abeida ${ }^{1}$ and Yahya S. H. Khraisat ${ }^{1,4}$ \\ ${ }^{I}$ Department of Electrical Engineering, Faculty of Engineering, Taif University, Taif, Kingdom of Saudi Arabia. \\ ${ }^{2}$ Department of Electrical Engineering, Faculty of Energy Engineering, Aswan University, Aswan, Egypt. \\ ${ }^{3}$ Department of Industrial Engineering, Faculty of Engineering, Taif University, Taif, Kingdom of Saudi Arabia; \\ ${ }^{4}$ Department of Electrical and Electronics Engineering, Al-Balqa' Applied University/Al-huson University College, Irbid, Jordan.
}

ORCID: 0000-0002-9431-4195

\begin{abstract}
This work presents utilizing interdisciplinary advancements in different engineering fields to propose a new modified design form with upgraded capabilities of a dependable and cost effective wireless sensor module, working as a subsystem of remote monitoring system and some considerations regarding control issues. The module design is developed such that various sensors types (voltage, current, analog and digital) can be plugged in, it can transmit data wirelessly using radio, $\mathrm{Wi}$ Fi or GSM techniques, also, can be powered by rechargeable battery or using local AC source. The presented module design is developed and intended to be employed in smart universities as a solution for achieving safety against fire and humidity in libraries and laboratories and luxury in smart studying rooms.
\end{abstract}

Keywords: Wireless sensor network; Smart university; Monitoring system; Design.

\section{INTRODUCTION}

Recent interdisciplinary advancements in different engineering fields have opened new research horizons in various areas, including in the area of communications and related electronics and computing fields. In this work authors are focusing on utilizing these advancements to the field of remote monitoring system, to propose a new modified design form with upgraded capabilities of a dependable at low cost wireless sensor node (module) system, based on hardware design as a subsystem of remote monitoring system. The suggested wireless sensor module system design is to be developed such that various sensors types (voltage, current, analog and digital) can be plugged in, it can be powered by rechargeable battery or using local AC source, transmit data wirelessly using radio, $\mathrm{Wi}-\mathrm{Fi}$ or GSM techniques, in addition, the suggested module design can be applied in various remote monitoring system application fields include logistic, healthcare, smart universities, environment monitoring and agriculture. In this work we are most interested in the employing remote wireless monitoring system in smart universities. The suggested wireless sensor module design, is to be developed as a subsystem of the remote wireless monitoring system, and intended to be employed in smart universities as dependable at low cost solution in achieving safety against fire and humidity (in libraries and laboratories) and luxury (in smart studying rooms with environment monitory for optimal climatic conditions).

The main goal of utilizing wireless communication systems is to overcome the need for any connection medium like wires, cables or any physical medium, to permit the acquired data on one working site, to be transmitted wirelessly to another intended base station for further processing. In wireless sensor network, a sensor node, using different sensors, senses physical parameters from user's specific application area and sends the acquired data to the intended destination. It is required for the sensor node to be with such characteristics and requirements like; distributed sensing, energy efficient, autonomous, adaptive to environment, miniaturized and low-cost.

\subsection{Related works}

Wireless sensor node designs, utilizing its concepts for different application, were intensively studied, many different designs and prototypes were introduced, such research woks can be found in different sources including in [1-11].

The structure of the current work is built as follow: Section 2 provides system methodology and working principle. In section 3 , the hardware design, selection and integration issues of all subsystems and components; and in section 4, system prototyping issues, hardware integration design and pictorial representation are discussed. Finally, the conclusion and the future work are made and discussed.

\section{SYSTEM METHODOLOGY AND WORKING PRINCIPLE}

The suggested system design consists of two main parts; the first part is the wireless sensor module, and the second part is the base station. The system principle of the work is developed 
based on distributing specific number of wireless sensor modules on a specific university's facility like class room, laboratory or library, to read the environmental, security or other conditions state and variables, process collected reading data and transmit it all. The second part is the base station, to which all readings data of all sensor modules are transmitted for further processing, saving and decision making. The architecture of the whole system is shown in figure 1(a)

The architecture of the main two parts are almost identical, the differences are in the control algorithm, and memory storage capacity, where the base station can also be used as sensors module. The architecture of the suggested wireless sensor module design is shown in figure 1(b). The wireless sensor module can be considered as consisting of the next main subsystems and components; control unit with algorithm for data processing, sensors subsystem, communication subsystem, interfaces, signal processing and power supply subsystem, and finally mechanical subsystem. To both modules, addition hardware components can be included like location finder unit. In the next sections for each of this subsystem, hardware design is to be suggested and selected.

The number of sensors embedded in one wireless sensors module, is limited only by the selected microcontroller, its input pins and their types. The reading of each and all sensors and peripherals are controlled by the control algorithm, a delay will be applied (program waits a finite time e.g. $5 \mathrm{~ms}$ ) to separate each reading from each sensor on the module, each acquired value is saved in a specific matrix location, when the values of all sensors are read and saved to matrix location, the data is transmitted using transceiver radio module, Wi-Fi module, or GSM Module, to the base station, where, the received data is acquired and saved with applied delay, then the saved data is processed and decisions are made, in addition, displaying real-time numerical data and graphical data plotting. To meet human requirement, data will be updated every 1 minute.

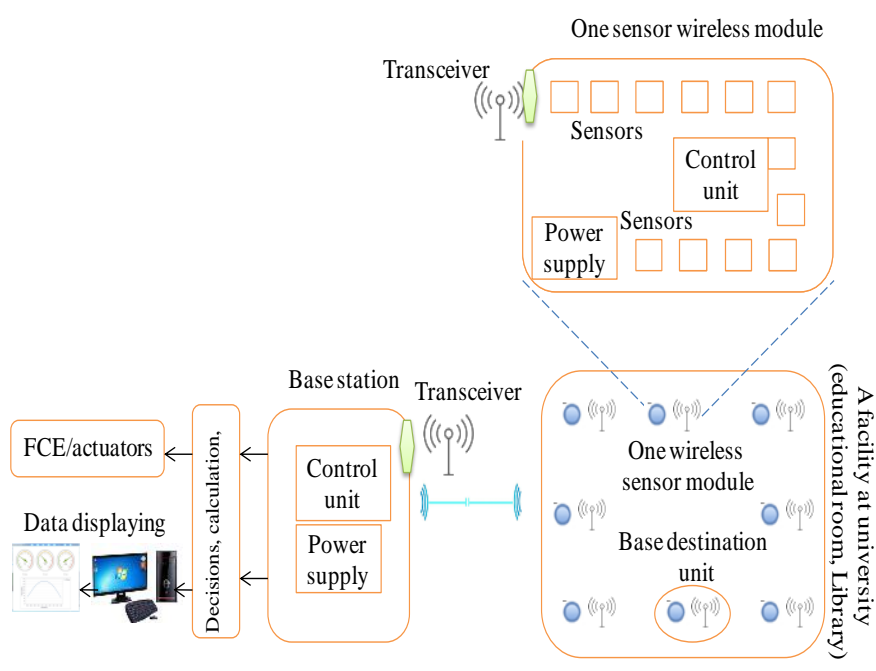

(a)

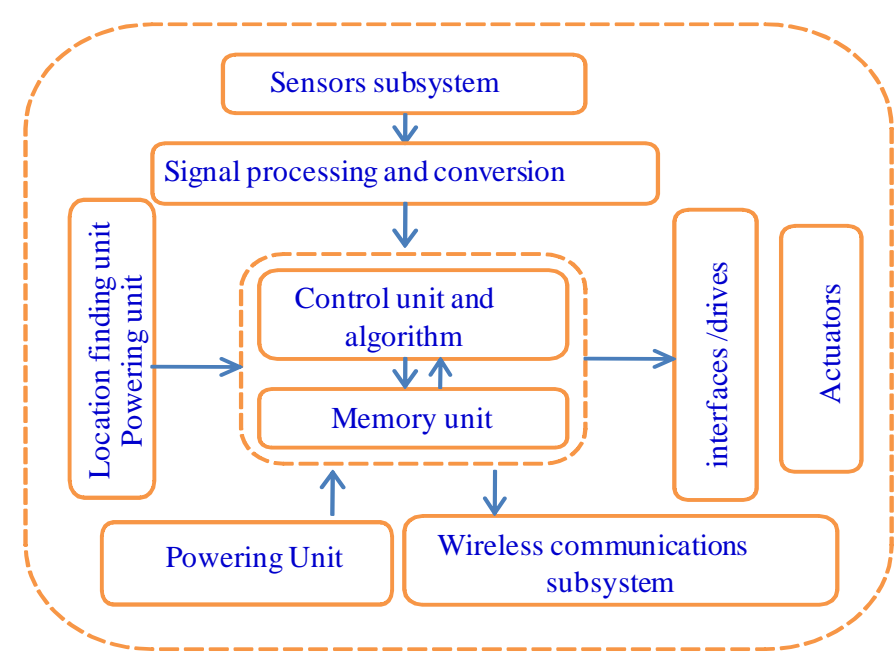

(b)

Figure 1. The suggested system design: (a). The suggested overall system design consisting of the two main parts wireless sensor module and base station. (b). The suggested architecture design of the wireless sensor module hardware design.

\section{THE HARDWARE DESIGN, SELECTION AND INTEGRATION ISSUES}

The system's main two parts, subsystem and components are to be designed in the next subsections as explained in section 2 .

\subsection{The control unit/algorithm design, selection and integration issues.}

Different types of control units are utilized in wireless sensor node design, including Microcomputer, Digital Signal Processors (DSP), Application-Specific Integrated Circuits (ASICs), and Field-Programmer Gate Array (FPGA). Due to its low power consumption, small size, acceptable signal processing capabilities and others, a suitable choice for the present system design is the developed based on Atmel SAM3X8E ARM Cortex-M3 CPU Arduino due board. This microcontroller comes with the following features; It is a $32-$ bit ARM core microcontroller, that is developed with 54 digital input/output pins (12 pins with PWM outputs), 12 analog inputs, 4 UARTs (hardware serial ports), an $84 \mathrm{MHz}$ clock [12, 13].

\subsubsection{Control Algorithm design and representation}

Figure 2, shows the flowchart representation of the control algorithm program for the wireless sensor module. 


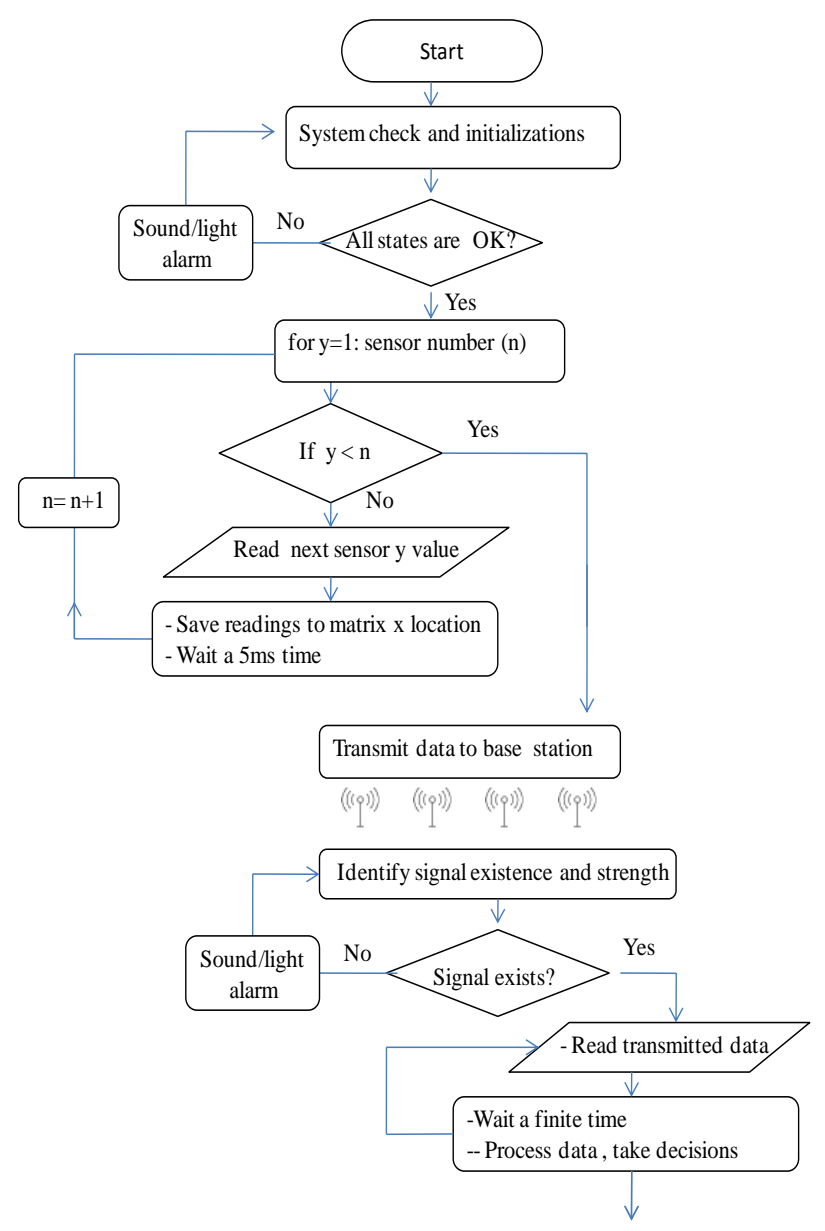

Figure 2. Algorithm flowchart representation.

\subsection{Wireless communication subsystem hardware design}

The main goal of utilizing wireless communication systems is to overcome the need for any connection medium like wires, cables or any physical medium, to permit the acquired data on one working site, to be transmitted wirelessly to another intended base station destination for further processing. There are different wireless mediums that can be utilized for communications in terms of data transmission and receiving, particularly: radio frequency, optical communications (laser), infrared, and ultrasonic. A appropriate communication method for the wireless module system design is the electromagnetic frequency, due to its ease of use, integrality with other hardware devices, long range communication and ability to penetrate obstacles like walls. In this context, there are different communications module tools that can be applied in the suggested wireless sensor design, including: a) ZigBee module, it is fit for application with low range (up to $30 \mathrm{~m}$ ) like indoor environments, low power consumption and data low transmission rate. b) Low Wi-Fi module, in comparison ZigBee module, it offers longer range and higher data transmission rate, but it consumes more power. c) Bluetooth, is suitable for application with a very low range $(10 \mathrm{~m})$ and low power consumption. d) Cellular communication mobile telephone system.

The suggested design is developed with capability to transmit data locally, within given space utilizing transceiver radio module or globally via internet utilizing Wi-Fi module, or GSM Module. A suitable hardware choice for radio communication is the shown in figure 3(a), NRF24L01 module with PA LNA SMA Antenna use $2.4 \mathrm{GHz}$ transceiver up to one KM distance. A appropriate hardware choice for Internet of Things (IoT) is utilizing the ESP8266 Wi-Fi module shown in figure 3(b), as alternative GSM Module is the shown in figure 3(c)
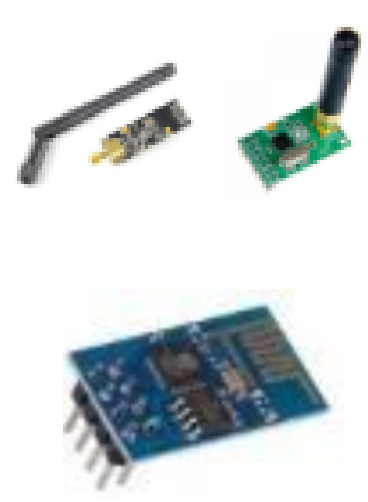

(b)

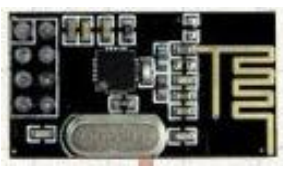

(a)

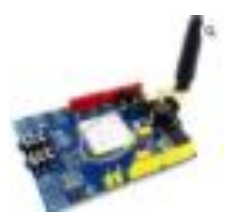

(c)
Figure 3. Wireless communication hardware choices : (a) Transceiver NRF24L01 module options. (b)The ESP8266 WiFi module. (c) SIM900 GSM Module.

\subsection{Sensors subsystem hardware design}

The wireless sensors module, is to be developed to accept two types of sensors output signals; with a voltage range between ( 0 to $5 \mathrm{VDC}$ ) a current between ( 4 to $20 \mathrm{~mA}$ ). For application in smart universities as a solution to help in achieving safety against fire and humidity (in libraries and laboratories) and luxury (in smart studying rooms with environment monitory for optimal climatic conditions). Different types of sensors can be utilized in the suggested wireless module design, including: temperature, humidity, light levels, dust levels pressure and many others.

Examples on such sensors include: the small sized atmospheric BME280 sensor to measure barometric pressure, humidity, and temperature readings, shown in figure 4(a); an alternative sensor is the shown in figure 4(b), DHT11 Temperature and Humidity. Other examples include, Grove Temperature and Humidity Sensor shown in figure 4(c) and the LM393 Light Sensor Module, as shown in figure 4(d).

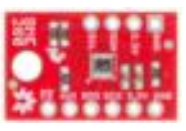

(a)

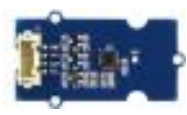

(c)

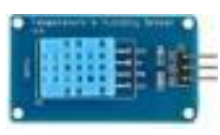

(b)

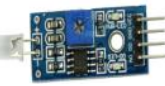

(d)
Figure 4. Sensors hardware choices : (a) Atmospheric BME280sensor. (b) DHT11 Temperature and Humidity. (c) Grove Temp. and Humidity Sensor. (d) LM393 Light Sensor Module. 


\subsection{Power supply, signal processing and interfaces design}

\subsubsection{Power management and supply unit design}

The power management and supply unit is responsible for powering the whole module, including for computations, data storage in memory, data transmission and HMI; there are different options to design this subsystem, the first and second options are applicable when sensor module is to be located for long time or permanently in specific location with nearby $\mathrm{AC}$ source. In this setup, the first option is using step down (220VAC to 5VDC) transformer power supply module (figure $5(\mathrm{a})$ ), which will supply the whole module with the needed power. The second option is using any of the shown in figure 5 (b) and 5(c) step down (AC $220 \mathrm{~V}$ to $5 \mathrm{~V}$ DC) power supply modules. The third option is suitable for autonomous wireless sensor module, using rechargeable (DC 3.7V 1000mAh) Lithium ion battery, shown in figure 5(d), to supply the whole module with the needed power. For charging the lithium batteries, TP4056 Lithium battery charger and protection module shown in figure 5(e) is utilized. The wiring of charging unit with battery is shown in figure 5(f).

Based on these three options, a suggested charging unit design developed and is presented in figure $5(\mathrm{~g})$. This charging module will be applied in the suggested wireless sensor module design, as a power management and supply unit. The 220VAC to 5VDC transformer, shown in figure 5(a), can be an integrated part of the wireless sensor module design, it can be placed inside the sensor module housing, that in turn has slot for wiring and plugging the AC source, or it can be used traditionally with plug in for AC powering.

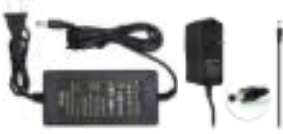

(a)

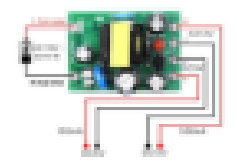

(c)

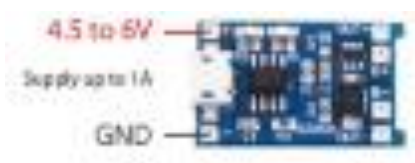

(e)

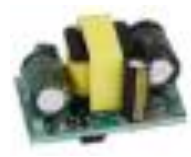

(b)

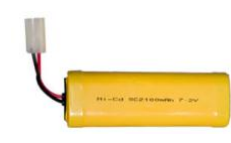

(d)

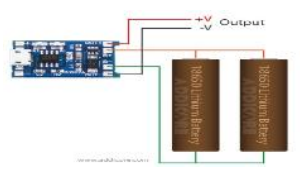

(f)

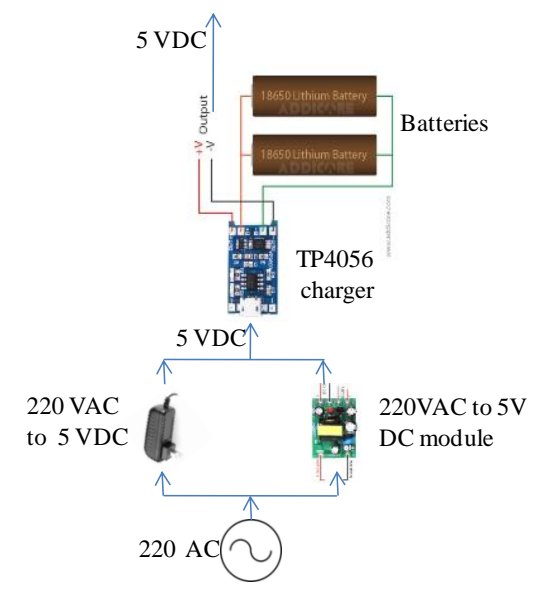

(g)

Figure 5. Power management and supply hardware: (a) AC $220 \mathrm{~V}$ to $5 \mathrm{~V}$ DC step down power module. (b) AC $220 \mathrm{~V}$ to $5 \mathrm{~V}$ DC step down power supply module. (c) AC $220 \mathrm{~V}$ to $5 \mathrm{~V}$ DC step down power supply module.. (d) Rechargeable battery. (e) TP4056 Lithium battery charger and protection Module. (f) The battery charging module. (g) Pictorial diagram of the suggested charging unit module design.

\subsubsection{Interfaces design}

Interfaces can be divided into two types; the human machine interface (HMI) and the interfaces between hardware components.

The HMI design is the design for data inputting/outputting to and from the system. For the current design, the HMI include the following; a) Medium for data transfer to $\mathrm{PC}$, for programming the microcontroller and for charging the batteries, a suitable choice is the FT232RL USB module shown in figure 6(a), this module is to be wired to programming USB port on Arduino due board. b) Light (LED) and sound (buzzer) indicators can be utilized for notifications and indications, suitable choices are the LED and buzzer shown in figure 6(b). For data massage displaying, and debugging purposes, a 16X2 LCD module shown in figure 6(c) is a good choice. An ON/OFF switch for switching ON/OFF the module is shown in figure 6(d). The hardware interfaces design is A Plug-in Point for power supply and charging batteries, can be developed using one female /male pair of DC Power connectors $2.1 \times 5.5 \mathrm{~mm}$ shown in figure 6(e), or DC power jack shown in figure $6(\mathrm{f})$. For wiring the sensors to the wireless module, screw terminal blocks, shown in figure $6(\mathrm{~g})$, are utilized. Industrial field transmitter (sensors), for signal output, use (4 to $20 \mathrm{~mA}$ ) current loop. In case the current based sensors to be utilized in the monitoring system design, there are different options for converting the output 4 to $20 \mathrm{~mA}$ to voltage (0 to $5 \mathrm{VDC}$ ). one option is as shown in figure $6(\mathrm{~h})$, placing a parallel between sensor's output signal and ground. Further software integration is applied to convert, by mapping, the resulted voltage from (1 to 5 VDC) to (0 to 5 VDC); another option is utilizing current to voltage converter module shown in Figure 6 (i). DS1302 real time clock (RTC) module shown in figure 6(i), can be utilized for time references and calculations, this RTC module contains 
a real-time clock and calendar. For providing extra memory for base station module for data saving and processing, the shown in figure $6(\mathrm{k})$, micro SD card module TF card memory shield is selected. Transistor 2N2222 is selected as drive to control the light and sound indicators' operation; alternatives to this transistor can be BC549, BC636 and BC639. The circuit for driving the light and sound indicator is shown in figure 6(1)

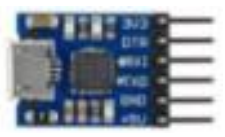

(a)

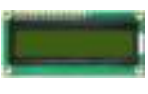

(c)

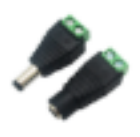

(e)

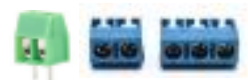

(g)

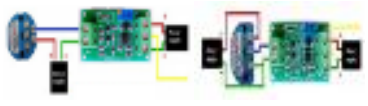

(i)

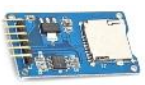

(k)

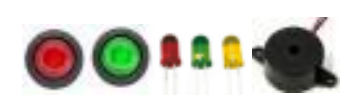

(b)

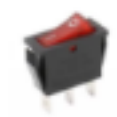

(d)

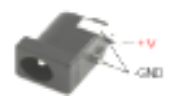

(f)

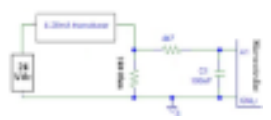

(h)

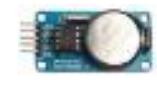

(j)

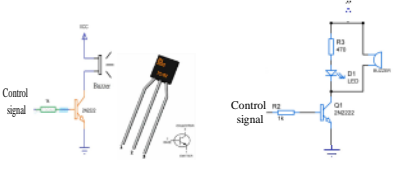

(1)
Figure 6. Human machine interface design: (a) USB module as Medium for data transfer from/to PC and programming; (b) Light (LED) and sound (buzzer) indicators con be utilized for notifications; (c) 16X2 LCD module for data massage; (d) An ON/OFF switch; (e) Female Male pair of DC Power connectors; (f) DC power jack; (g) Screw terminal block for wiring sensor to module; (h) Circuit for converting sensor's signal (4to 20mA) Into (1to5 VDC); (i) Current to voltage converter for 2\&3 wires sensor; (j) DS1302 Real Time Clock Module; (k) Micro SD card module memory shield; (l) Transistor 2N2222, and drive circuit to control light/sound indicators.

\subsection{The mechanical subsystem design and integration issues}

The mechanical subsystem for the suggested wireless sensor module design is minimized to the structure (housing), its materials and dimensions. Two versions of suggested housing design for the wireless sensor module, with hardware integration and placement of components in overall system housing is shown in figure 7(a) and 7(b). The same housing design is applicable for the wireless final base station, the screw terminal block can be utilized to wire interface circuits for controlling actuators.

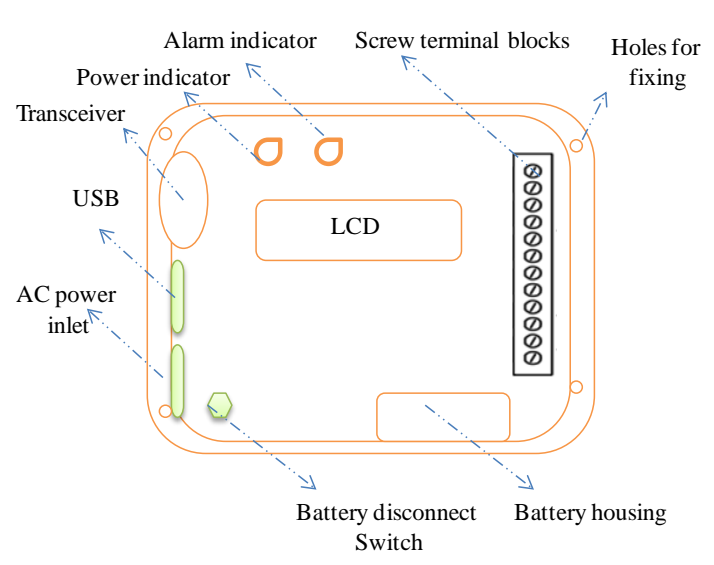

(a)

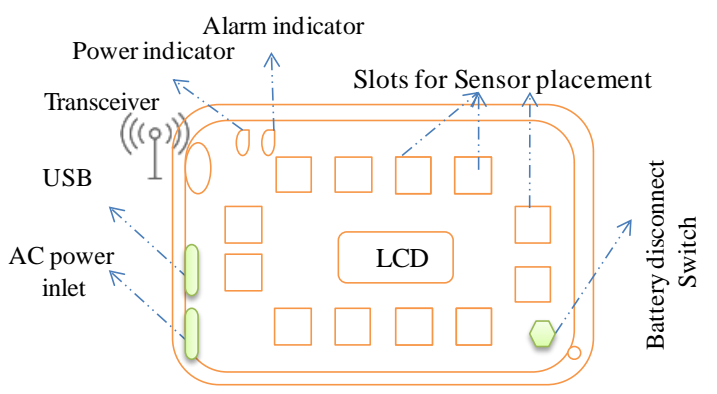

(b)

Figure 7. The suggested housing design: (a) Version1; (b) Version 2.

\section{SYSTEM PROTOTYPING; HARDWARE INTEGRATION DESIGN AND PICTORIAL REPRESENTATION.}

The functional components diagram of the wireless sensors module is shown in figure 8(a). The suggested wireless sensor module design with the designed and selected subsystems, hardware components, circuits and modules are to be integrated to produce the overall one system, the hardware integration and placement of components in overall system design and housing is shown in Figure 8(b). 


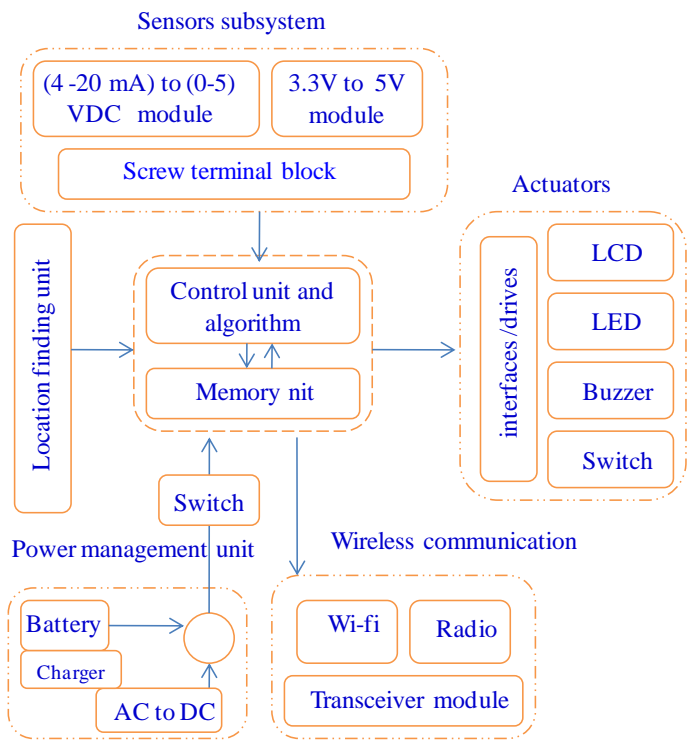

(a)

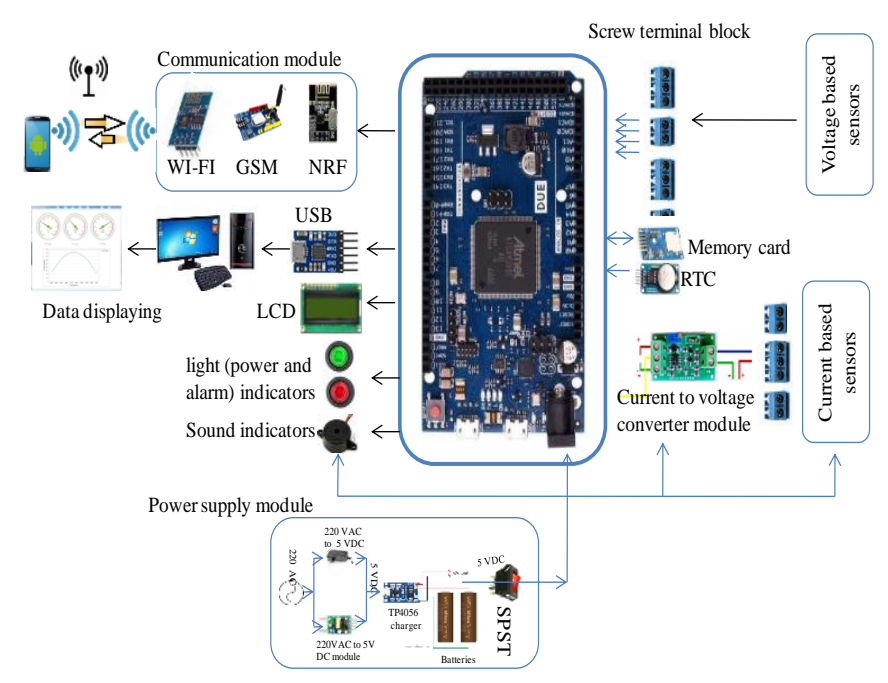

(b)

Figure 8. hardware integration design and pictorial representation: (a) The functional components wireless sensor module hardware design. (b) The hardware integration of all hardware physical components in one system prototype pictorial representation.

\section{CONCLUSIONS}

In this work, dependable and cost effective wireless sensor module was designed and developed, the proposed new design was improved to have the capability of plugging in deferent sensors types, also it simplifies transmitting the data wirelessly using radio, Wi-Fi or GSM techniques, also it has the advantages of being powered using rechargeable battery or local AC source. The proposed work is intended to be employed in smart universities as a solution for achieving safety against fire and humidity in libraries and laboratories and luxury in smart studying rooms.

\section{ACKNOWLEDGMENT}

This research was supported by Research Groups Program funded by Deanship of Scientific Research, Taif University, Ministry of Education, Kingdom of Saudi Arabia (grant number: 1-440-6140).

\section{REFERENCES}

[1] S. K. Nagpal and S. Sudha, "Design and development of a sensor node to monitor and detect change in internal parameters of a cold storage system," in 2015 Global Conference on Communication Technologies (GCCT), 2015, pp. 745-748.

[2] C. Suh and Y. Ko, "Design and implementation of intelligent home control systems based on active sensor networks," IEEE Transactions on Consumer Electronics, vol. 54, no. 3, pp. 1177-1184, 2008.

[3] W. Chung, C. Chen, and J. Chen, "Design and Implementation of Low Power Wireless Sensor System for Water Quality Monitoring," in 2011 5th International Conference on Bioinformatics and Biomedical Engineering, 2011, pp. 1-4.

[4] J. Zhang, G. Song, H. Wang, and T. Meng, "Design of a Wireless Sensor Network Based Monitoring System for Home Automation," in 2011 International Conference on Future Computer Sciences and Application, 2011, pp. 57-60.

[5] S. Macis et al., "Home telemonitoring of vital signs through a TV-based application for elderly patients," in 2015 IEEE International Symposium on Medical Measurements and Applications (MeMeA) Proceedings, 2015, pp. 169-174.

[6] J. M. G. Duarte, E. Cerqueira, and L. A. Villas, "Indoor patient monitoring through $\mathrm{Wi}-\mathrm{Fi}$ and mobile computing," in 2015 7th International Conference on New Technologies, Mobility and Security (NTMS), 2015, pp. 1-5.

[7] H. Jinsoo, L. Haeryong, and P. Kwang-Roh, "Remotecontrollable and energy-saving room architecture based on ZigBee communication," in 2009 Digest of Technical Papers International Conference on Consumer Electronics, 2009, pp. 1-2.

[8] Q. Huang, F. Zhang, and W. Chen, "Research and 
International Journal of Engineering Research and Technology. ISSN 0974-3154, Volume 13, Number 5 (2020), pp. 909-915

○ International Research Publication House. https://dx.doi.org/10.37624/IJERT/13.5.2020.909-915

Realization of Blood Pressure Monitoring System Based on ZigBee Wireless Sensor Network," Berlin, Heidelberg, 2012, pp. 205-211: Springer Berlin Heidelberg.

[9] J. G. L. K. S. Kim, S. S. Ahn, and J. Lee, "A Study on USNMulti- Modal Sensor Fusion Technique for Intelligent Air Conversions Digital Home Appliances," MITA, pp. 172 - 175, 2010.

[10] R. S. Dilmaghani, H. Bobarshad, M. Ghavami, S. Choobkar, and C. Wolfe, "Wireless Sensor Networks for Monitoring Physiological Signals of Multiple Patients," IEEE Transactions on Biomedical Circuits and Systems, vol. 5, no. 4, pp. 347-356, 2011.

[11] S. Purnima Puneet, "Zigbee and GSM based patient health monitoring system," in 2014 International Conference on Electronics and Communication Systems (ICECS), 2014, pp. 1-5.

[12] T. N. May Thu Win, Nay Win Zaw, "Design and Implementation of Elevator Control System by Arduino Microcontroller," International Journal of Science, Engineering and Technology Research (IJSETR), vol. 7, no. 12, 2018.

[13] R. Y. M. Li, H. Li, C. K. Mak, and T. Tang, "Sustainable Smart Home and Home Automation: Big Data Analytics Approach," International Journal of Smart Home, vol. 10, pp. 177-198, 09/10 2016. 